Supporting Information

\title{
DC Voltage Modulation for Integrated Self-Charging Power Systems of Triboelectric Nanogenerators and Ion Gel/ $/ \mathrm{WO}_{3}$
}

\section{Supercapacitors}

Ju Hyun Lee ${ }^{1}$, Keon-Woo Kim ${ }^{1}$, Jin Kon Kim ${ }^{1 *}$, and Unyong Jeong ${ }^{2 *}$

${ }^{1}$ Department of Chemical Engineering, Pohang University of Science and Technology, Pohang 37673, Republic of Korea

*jkkim@postech.ac.kr

${ }^{2}$ Department of Materials Science and Engineering, Pohang University of Science and Technology, Pohang 37673, Republic of Korea

*ujeong@ postech.ac.kr

Keywords

triboelectric nanogenerator, supercapacitor, ion gel, tungsten oxide, charge storage 


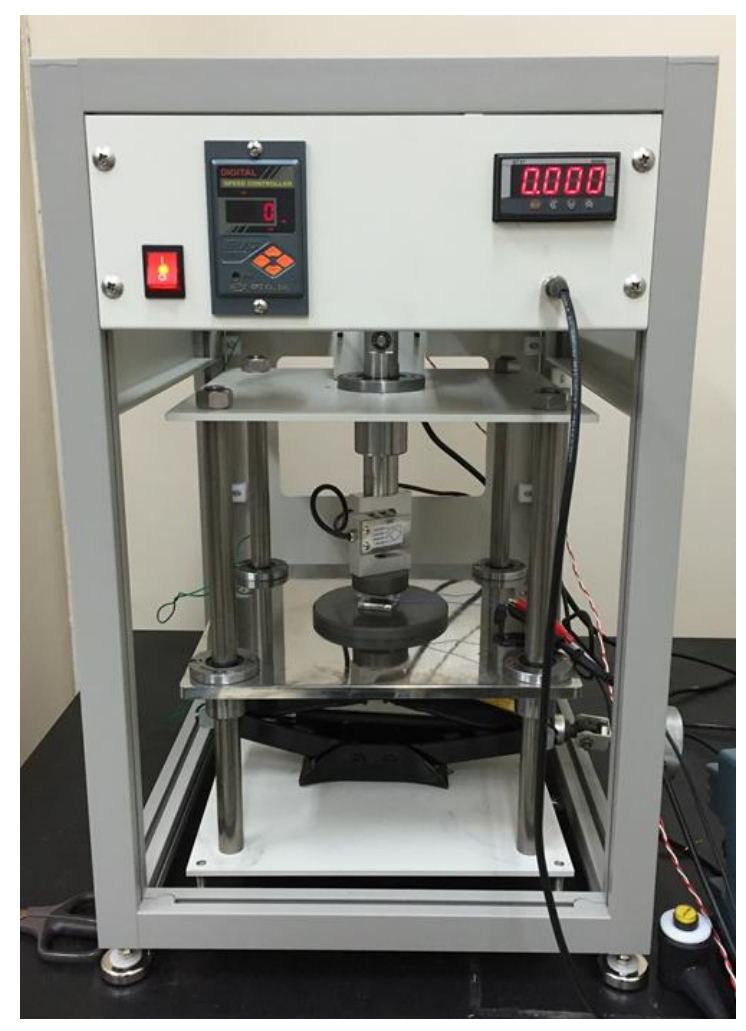

Figure S1. A home-made contact machine for applying vertical force on TENG device. The sample is located between gray metal substrates where the top substrate oscillates vertically. 


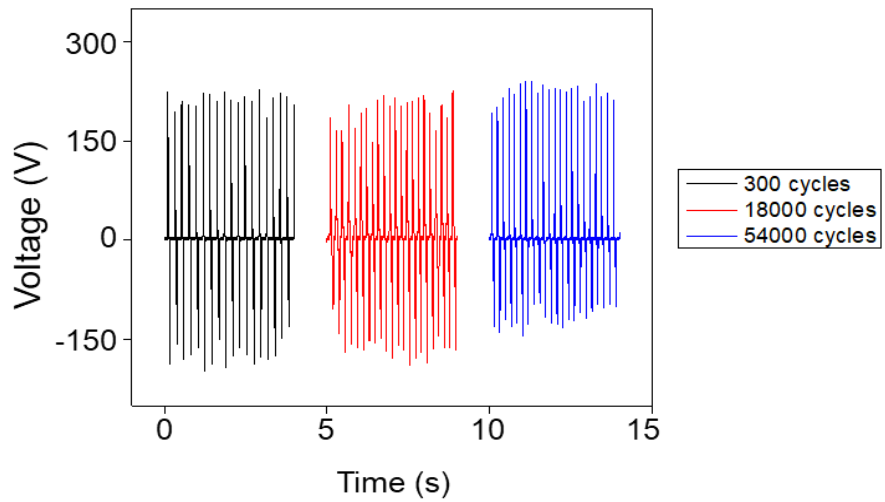

Figure S2. The voltage profiles after cycle tests $(300,18000,54000$ cycles) of the fabricated TENG generating peak-to-peak voltage of $388 \mathrm{~V}, 384 \mathrm{~V}, 358 \mathrm{~V}$, respectively. 


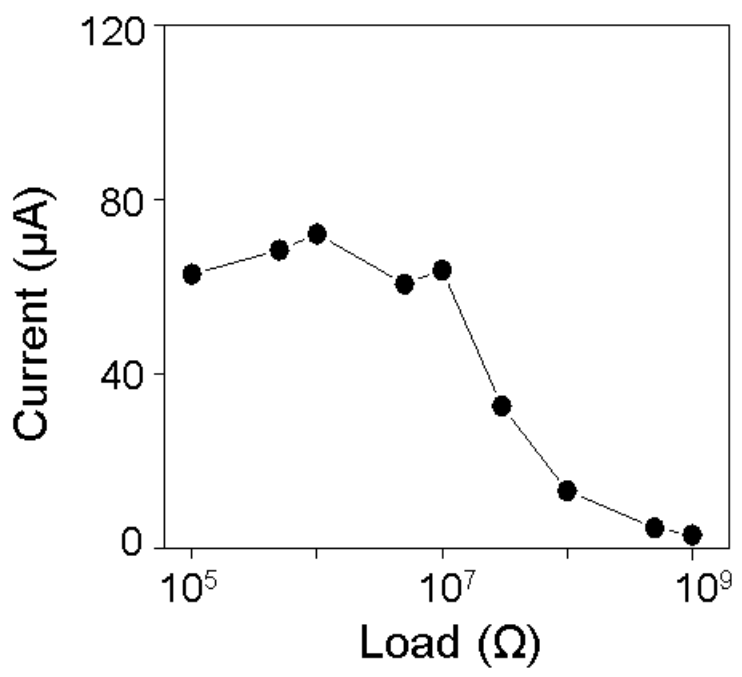

Figure S3. Peak-to-peak output current according to the load resistance. 


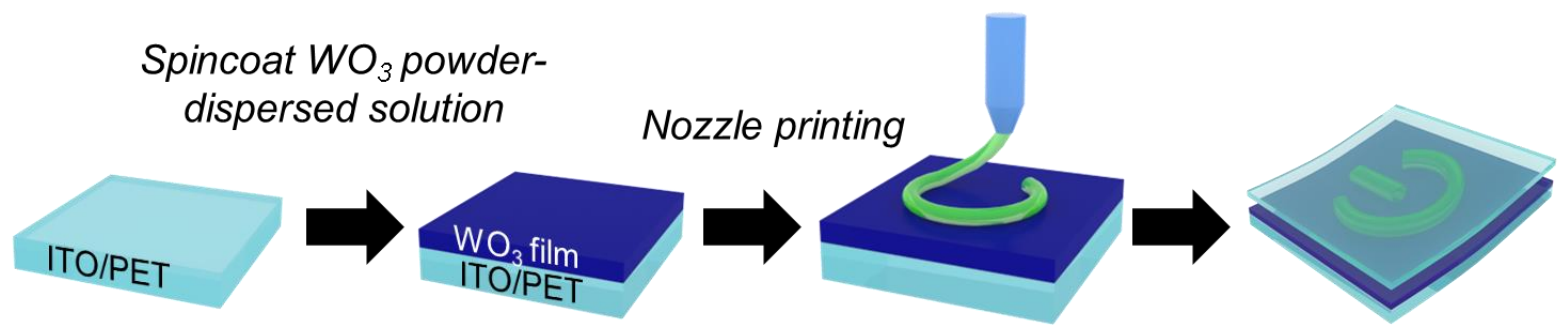

Figure S4. Schematic of the fabrication process of the Gel- $\mathrm{WO}_{3}$ supercapacitor. 


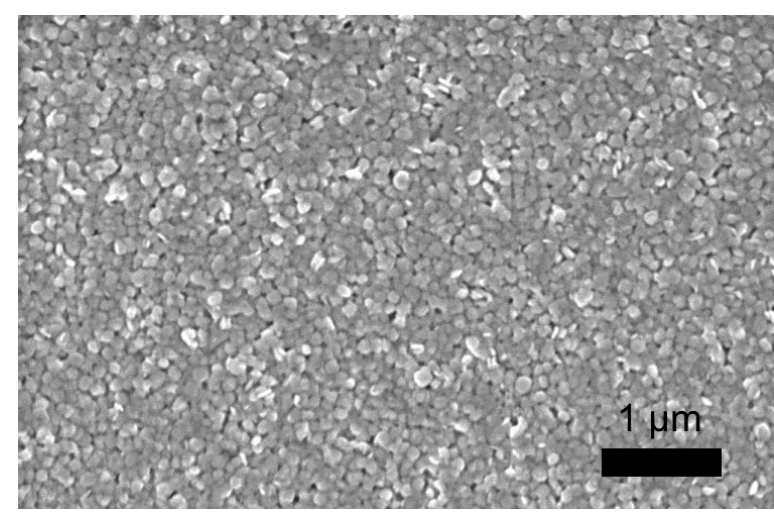

Figure S5. SEM image of the fabricated $\mathrm{WO}_{3}$ film on ITO/PET exhibiting a porous structure composed of nanoparticles. 

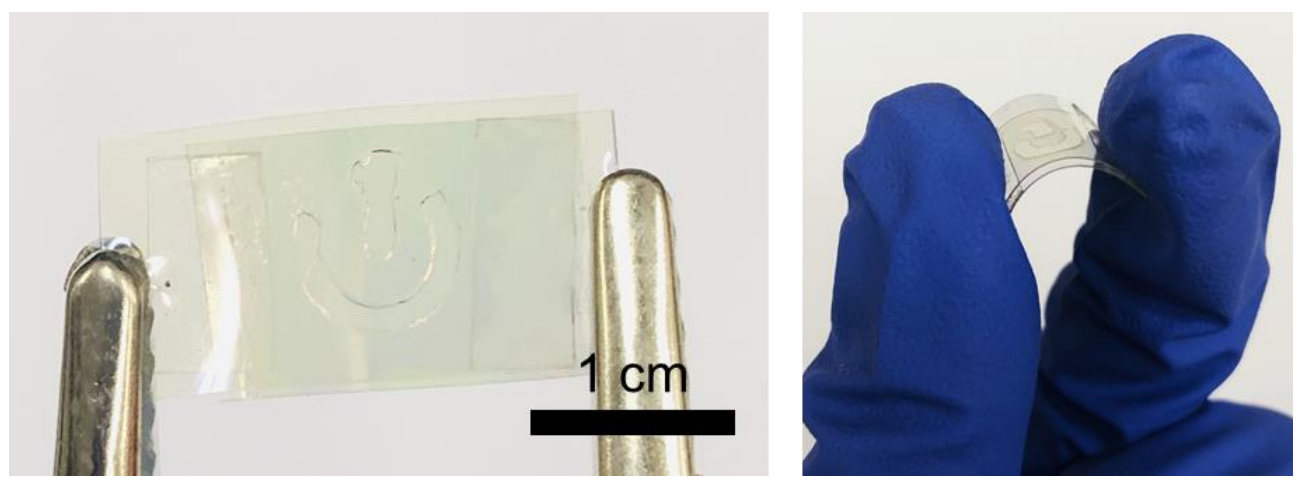

Figure S6. Photograph of Gel- $\mathrm{WO}_{3}$ device lifted with metal clips showing a transparent and flexible ion gel printed and sandwiched between two films of ITO/PET. 


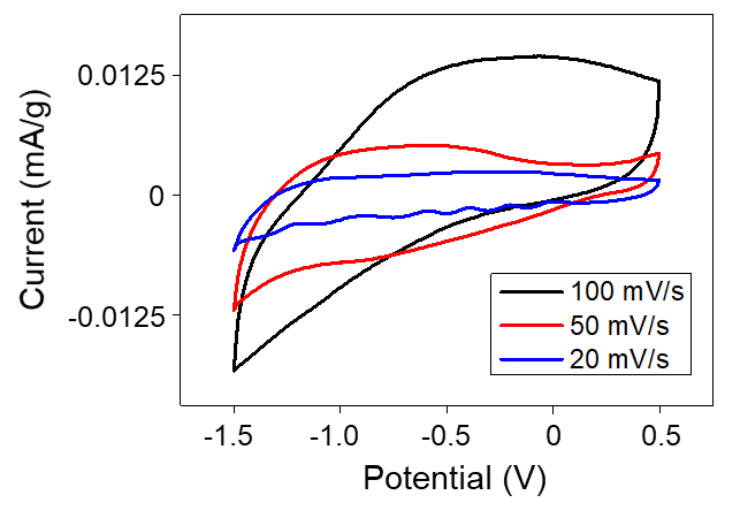

Figure S7. The cyclic voltammetry data of Gel- $\mathrm{WO}_{3}$ supercapacitor between $-1.5 \mathrm{~V}$ to $0.5 \mathrm{~V}$ at voltage scanning rate of $100,50,20 \mathrm{mV} / \mathrm{s}$. 


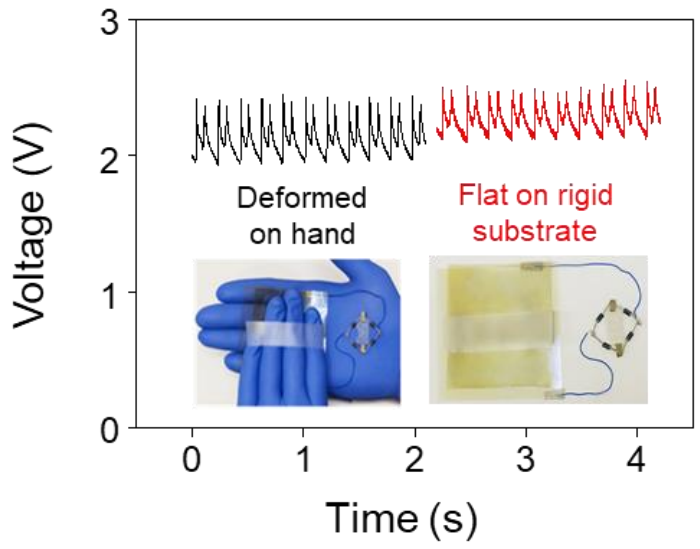

Figure S8. The output voltage of the integrated power system during deformed state on hand $(2.2 \mathrm{~V})$ and flat state on rigid substrate $(2.3 \mathrm{~V})$, as depicted in the inset images. 

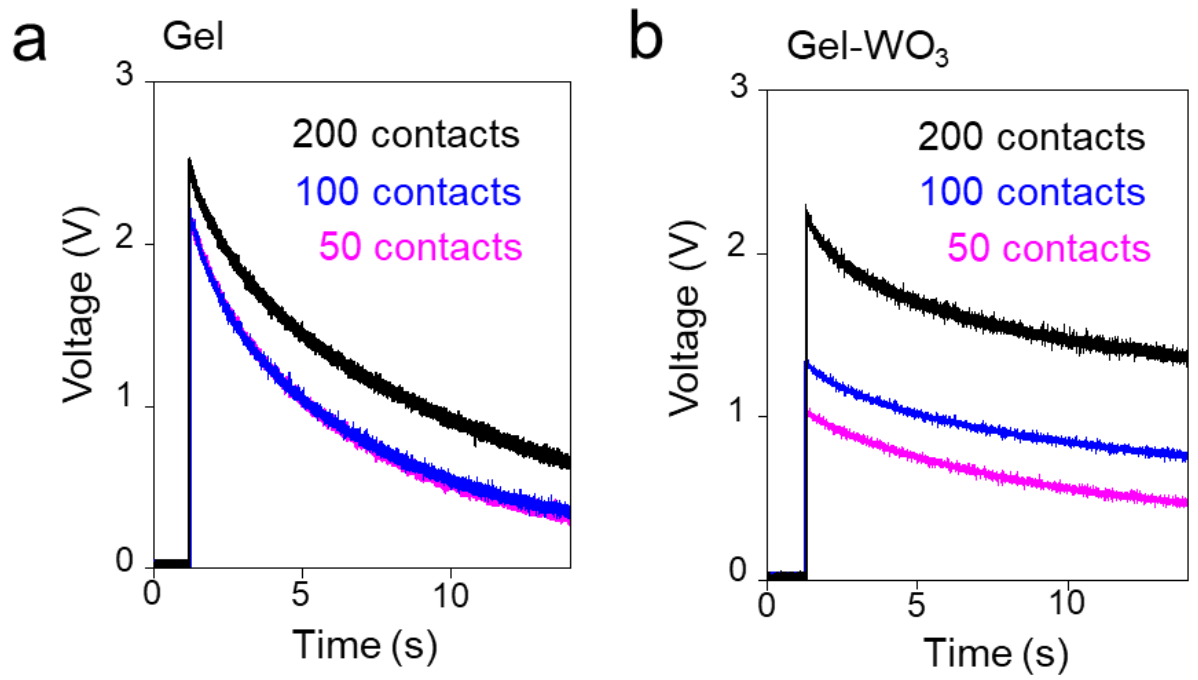

Figure S9. Relaxation profiles of (a) Gel and (b) Gel-WO 3 after 50 (pink), 100 (blue), and 200 (black) contacts applied on TENG, representing a fast formation of EDL in Gel and a gradual insertion of $\mathrm{Li}^{+}$in $\mathrm{WO}_{3}$ in Gel- $-\mathrm{WO}_{3}$. 

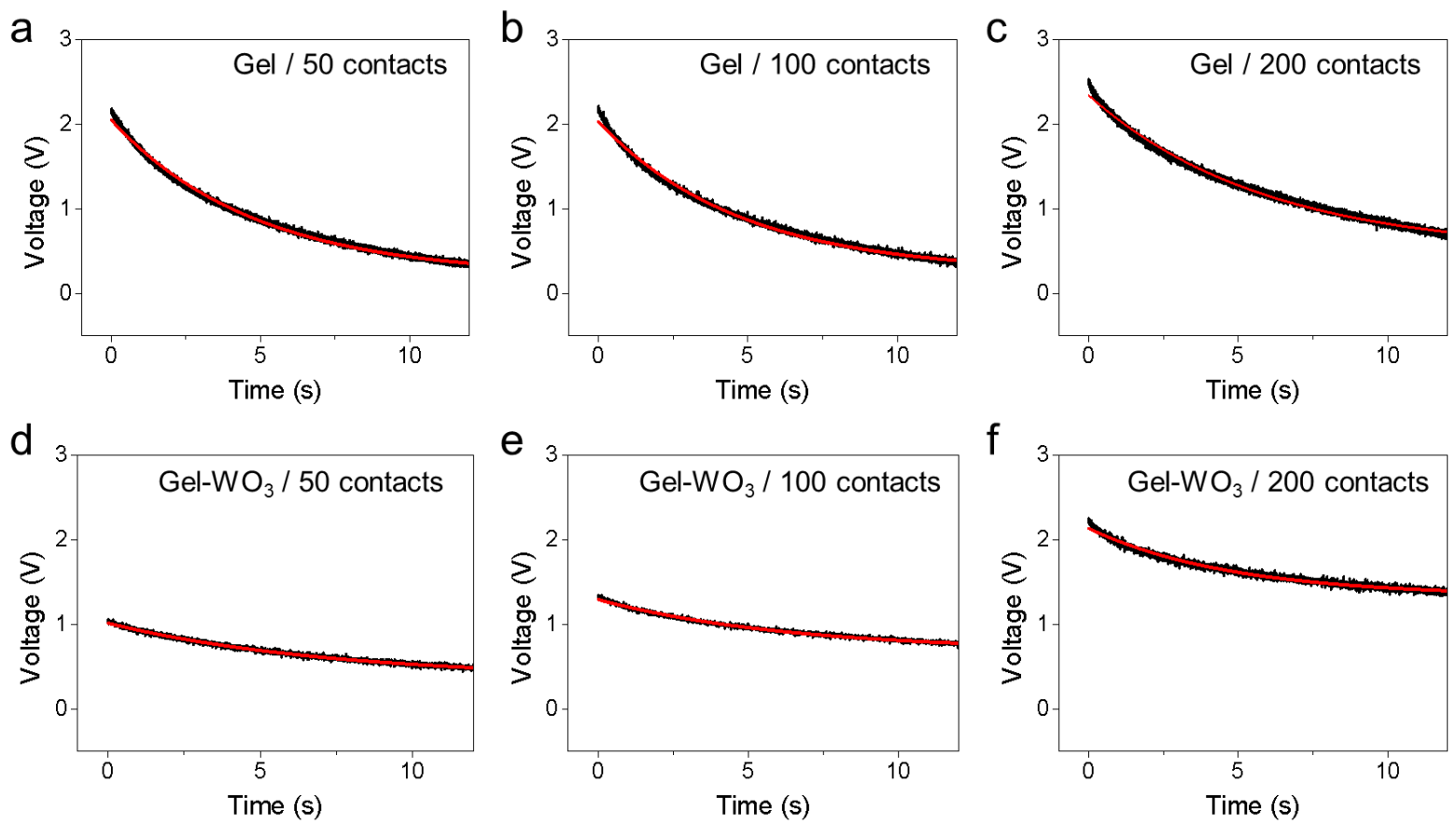

Figure S10. Voltage graphs of the exponential fitting lines (red) and the measured relaxation profile (black) for (a-c) Gel and (d-f) Gel-WO 3 after 50, 100, and 200 contacts applied on TENG.

Table S1. The fitting parameters for exponential fitting of the Gel.

\begin{tabular}{cccc}
\hline Contacts & $\boldsymbol{V}_{\text {offset }}[\mathbf{V}]$ & $\boldsymbol{V}_{\mathbf{0}}[\mathbf{V}]$ & $\boldsymbol{\tau}[\mathbf{s}]$ \\
\hline 50 & 0.2 & 1.8 & 4.8 \\
100 & 0.3 & 1.8 & 4.7 \\
200 & 0.5 & 1.9 & 5.8 \\
\hline
\end{tabular}

Table S2. The fitting parameters for exponential fitting of the Gel- $\mathrm{WO}_{3}$.

\begin{tabular}{cccc}
\hline Contacts & $\boldsymbol{V}_{\text {offset }}[\mathbf{V}]$ & $\boldsymbol{V}_{\mathbf{0}}[\mathbf{V}]$ & $\boldsymbol{\tau}[\mathbf{s}]$ \\
\hline 50 & 0.4 & 0.7 & 7.4 \\
100 & 0.7 & 0.6 & 6.0 \\
200 & 1.3 & 0.8 & 4.7 \\
\hline
\end{tabular}



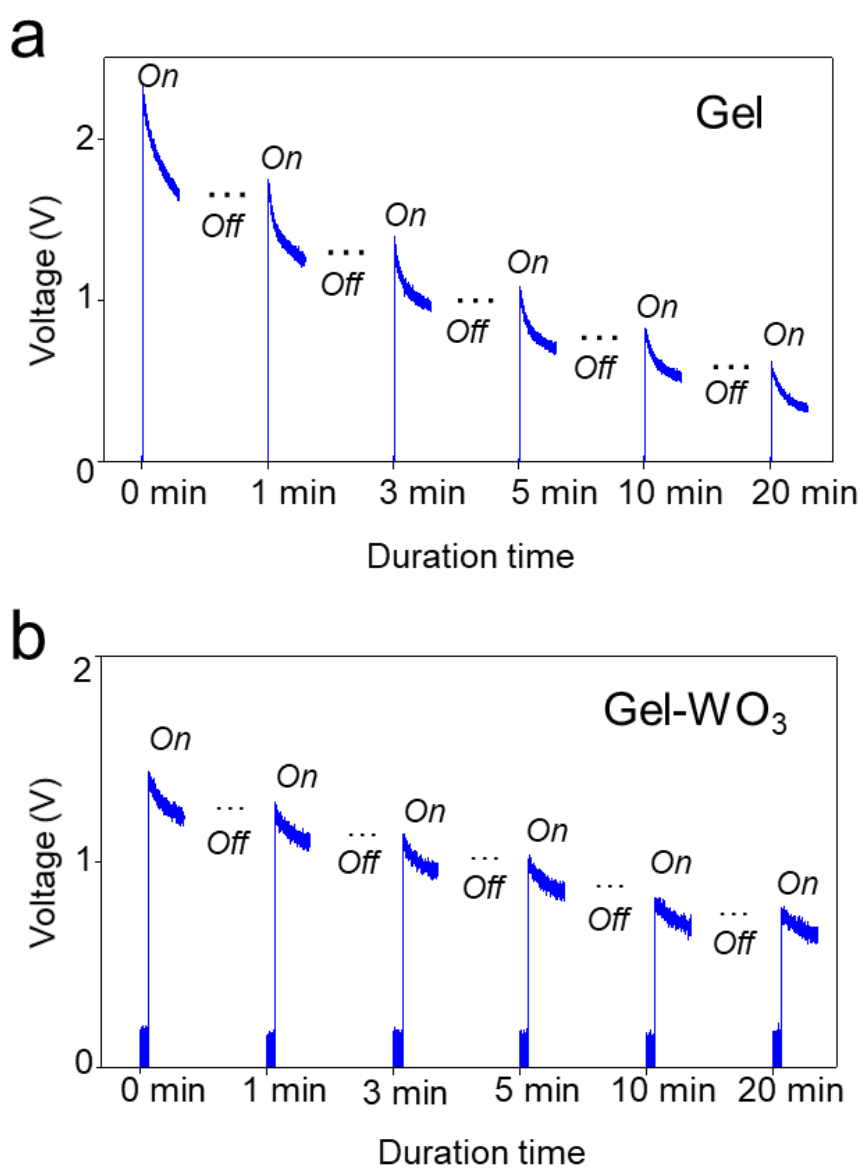

Figure S11. Voltage profiles of (a) the Gel and (b) the Gel- $\mathrm{WO}_{3}$ after 100 contacts applied, while the oscilloscope was connected (measurement on) and disconnected (measurement off) repeatedly. 
Table S3. The fitting parameters for equivalent circuit of the Gel.

\begin{tabular}{ccccc}
\hline Contacts & $\boldsymbol{R}_{\mathbf{e}}[\boldsymbol{\Omega}]$ & $\boldsymbol{R}_{\mathrm{dl}}[\mathbf{M} \boldsymbol{\Omega}]$ & $\boldsymbol{C}_{\mathbf{d l}}[\boldsymbol{\mu F}]$ & $\boldsymbol{n}_{\mathbf{1}}$ \\
\hline 50 & 317 & 38 & 0.24 & 0.88 \\
100 & 319 & 20 & 0.22 & 0.88 \\
200 & 325 & 12 & 0.26 & 0.88 \\
\hline
\end{tabular}

Table S4. The fitting parameters for equivalent circuit of the Gel- $\mathrm{WO}_{3}$.

\begin{tabular}{cccccccc}
\hline Contacts & $\boldsymbol{R e}_{\mathrm{e}}[\mathbf{\Omega}]$ & $\boldsymbol{R}_{\mathrm{dl}}[\mathrm{M} \boldsymbol{\Omega}]$ & $\boldsymbol{C}_{\mathrm{dl}}[\boldsymbol{\mu F}]$ & $\boldsymbol{n}_{1}$ & $\boldsymbol{R w 0 3}[\mathrm{k} \boldsymbol{\Omega}]$ & $\boldsymbol{C w O 3}_{\mathrm{w}}[\boldsymbol{\mu F}]$ & $\boldsymbol{n}_{2}$ \\
\hline 50 & 857 & 330 & 0.82 & 0.87 & 8.5 & 2.96 & 0.81 \\
100 & 719 & 30 & 0.61 & 0.86 & 6.9 & 2.29 & 0.89 \\
200 & 694 & 23 & 0.58 & 0.86 & 3.7 & 1.99 & 0.95 \\
\hline
\end{tabular}

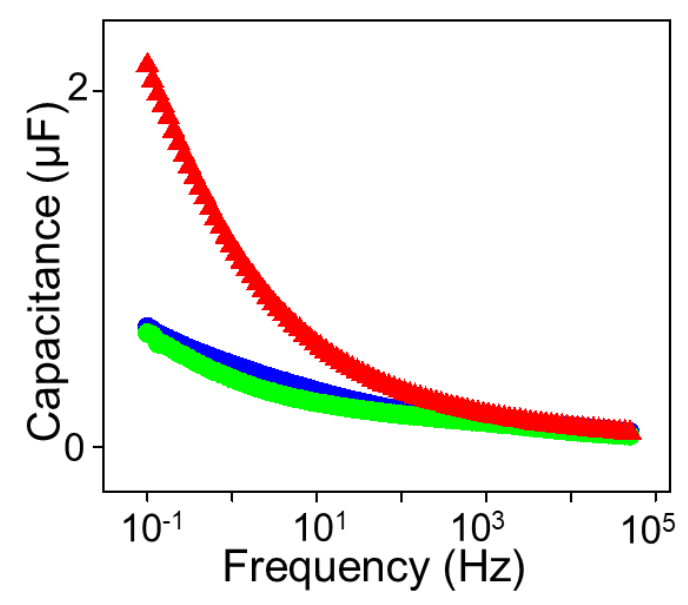

Figure S12. Measured capacitance of Gel- $\mathrm{WO}_{3}$ supercapacitor before (red) and after applied DC voltage of $2 \mathrm{~V}$ by a function generator (green), and after 200 contacts applied on TENG connected with Gel- $\mathrm{WO}_{3}$ (blue), illustrating the reduced capacitance of Gel-WO 3 after DC voltage was applied. 


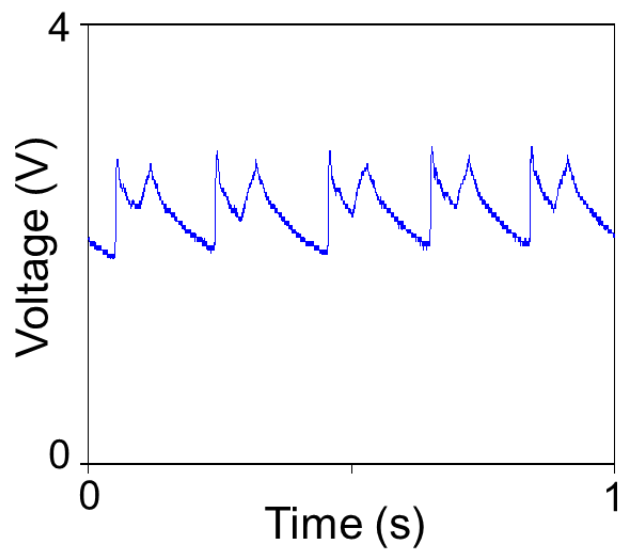

Figure S13. DC output voltage profile measured from the Gel capacitor $\left(V_{\mathrm{Gel}}\right)$ while the contact-separation was applied on TENG at $5 \mathrm{~Hz}$. 


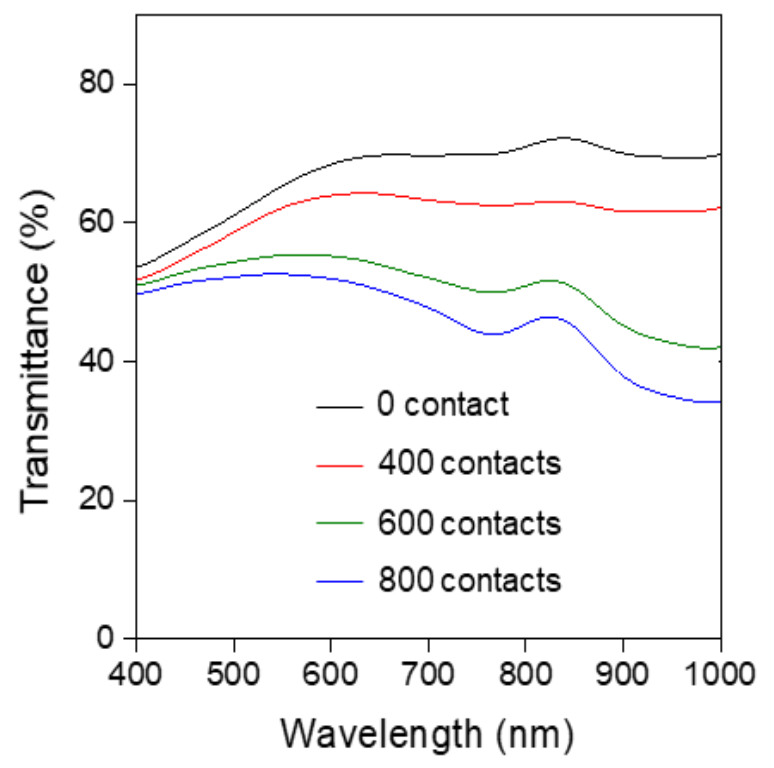

Figure S14. Transmittance of the printed ion $\mathrm{gel} / \mathrm{WO}_{3}$ supercapacitor depending on the number of contacts applied on TENG. 
Movie S1. Electrochromic coloration video (8x speed) during hand touch applied at $5 \mathrm{~Hz}$ on TENG connected with Gel-WO 3 supercapacitor. 